\title{
Importancia ecológico-económica del aprovechamiento de los bosques
}

\author{
Raymundo Dávalos Sotelo
}

\section{RESUMEN}

Satisfacer las necesidades de la población de una manera racional, perdurable y ecológicamente sensata es el principal reto de las sociedades modernas. En muchas de ellas, los recursos forestales han desempeñado un papel preponderante en el desarrollo de la civilización y la contribución de la industria forestal al producto interno bruto es significativa. Este no es el caso de México.

La hipótesis central de este trabajo es que el uso de la madera no es nocivo para el medio ambiente. La madera es uno de los materiales para construcción más benignos. Es un recurso natural renovable económicamente atractivo para usos estructurales y arquitectónicos. Los materiales no renovables emplean considerablemente más energía por unidad de producción que la madera.

La madera como material de construcción tiene grandes ventajas. Como aislante, la madera es más eficiente energéticamente. Su eficiencia estructural en términos de la energía necesaria para producirla es mayor. Los bosques en crecimiento activo son enormes almacenadores de carbono. Ningún otro material provee este servicio ecológico que reduce el efecto invernadero. Otro punto destacado es el papel del bosque como elemento biosférico que regula la hidrología y la biogeoquímica terrestre.

El secreto para obtener todos estos beneficios está en cultivar el bosque; utilizarlo para la producción de bienes de consumo, con un gasto energético reducido y hacerlo que crezca para que produzca más oxígeno, capture más bióxido de carbono y conserve el suelo al mismo tiempo.

PALABRAS CLAVE:

Industria forestal, desarrollo, usos estructurales, recursos renovables, gasto energético, bióxido de carbono.

\section{ABSTRACT}

Satisfying the needs of the population in a rational, permanent and ecologically sensible way is the greatest challenge facing modern societies. Forest resources have played a significant role in the development of civilization and have contributed significantly to national income. This is not the case in Mexico.

The central hypothesis of this paper is that the use of wood is not harmful to the environment. Wood is one of the most benign construction materials available. It is a natural, renewable resource that is economically attractive for structural and

1 Investigador. Departamento de Productos Forestales y Conservación de Bosques. Instituto de Ecología, A.C. Apdo. Postal 63. 91000 Xalapa, Ver. México.

Manuscrito recibido para su publicación el 6 de Junio de 1996 
architectural uses. Non-renewable materials use up a great deal more energy per production unit than wood.

Wood as a construction material has many advantages. As an insulating material, it is more energetically efficient. Its structural efficiency, in terms of the energy required to produce it, is greater. Growing forests are huge carbon sinks and no other material performs the ecological service of reducing the greenhouse effect. Another notable benefit is the role of the forest as an element of the biosphere that regulates hydrology and earth biogeochemistry.

The secret to reaping all of these benefits is to cultivate forests, to use them for the production of commodities with low energy expenditure and to let them grow so that they produce more oxygen, capture carbon dioxide and maintain forest soils intact.

KEY WORDS:

Forest industry, development, structural uses, renewable resources, energy expenditures, carbon dioxide.

\section{INTRODUCCION}

Satisfacer las necesidades de la población de una manera racional, perdurable y ecológicamente sensata es quizás, el principal reto de las sociedades modernas. Para la satisfacción de estas necesidades, los pueblos cuentan con diferentes recursos y con variadas formas de organización. En las sociedades occidentales (Norte América, Europa Occidental) y en Asia, los recursos forestales han desempeñado un papel preponderante en el desarrollo de la civilización. En muchas de estas naciones, la contribución de la industria forestal y la de los productos forestales al producto interno bruto es significativa.
Desafortunadamente, este no es el caso de México. En este trabajo se pretende hacer una contribución a la discusión que actualmente tiene lugar en nuestro país acerca de las estrategias de desarrollo. Mi hipótesis es que el desperdicio de los recursos forestales nacionales, ha tenido y tendrá, consecuencias funestas para el país. El objetivo que busco es ilustrar que un uso amplio, generalizado, pero eficiente y racional de estos recuros redundaría en enormes beneficios para México. Lo opuesto, que sería la continuación del abandono tradicional de nuestros bosques, implicará graves consecuencias económicas y ecológicas.

\section{DEBATE ACERCA DEL USO DE LOS RECURSOS NATURALES}

En la actualidad, el debate acerca del uso racional de los recursos naturales mundiales ha alcanzado niveles importantes en la opinión pública internacional. Prueba de ello es la reciente "Cumbre de la Tierra" en Río de Janeiro con sus consiguientes secuelas. En los países industrializados, el debate acerca del uso continuado de los recursos forestales influye de manera decisiva en la política interior de esos países y, por los niveles de producción y consumo involucrados, tiene efectos globales en la economía y el ambiente. Los grupos denominados "ecologistas" apoyan la tendencia general de disminuir el uso de los recursos naturales renovables locales sustituyéndolos con productos sintéticos 0 importados. En cambio, los industriales y académicos de esos países involucrados en el aprovechamiento del bosque argumentan que, si se desalienta el uso de los recursos forestales nativos, se tendrán serias consecuencias negativas.

Los argumentos principales se centran en el consumo energético y sus aspectos derivados. Todos los bienes y 
servicios, tanto económicos como ambientales, tienen costos de producción energéticos cuantificables, directos e indirectos (Cleveland et al., 1984). Los puntos centrales de la discusión aquí planteada se refieren a los costos energéticos de los recursos forestales comparados con otros materiales opcionales.

Como es de sobra conocido, existen fuertes corrientes de opinión en sentidos opuestos: la industria de los productos forestales por siempre se ha basado en el concepto de la capacidad de renovación del bosque, mientras que la influencia de los grupos ambientalistas que abogan por el cese del corte de árboles en los bosques crece continuamente. Lo que parece que no puede evitarse es que el uso de los recursos forestales se incremente a nivel mundial en el futuro, simplemente porque el crecimiento de la población generará mayor demanda sobre éstos y todos los tipos de recursos y no es sencillo ni deseable y mucho menos económico encontrar sustitutos adecuados a los productos que provienen de bosque. Ignorar los argumentos de cualquiera de los dos bandos sin someterlos a un profundo análisis puede provocar consecuencias graves e irreversibles ya sea a los niveles de vida de la población $o$ al medio ambiente mismo.

\section{NECESIDAD DE SATISFACTORES PROVENIENTES DEL BOSQUE}

El crecimiento de la población tiene una relevancia fundamental en cualquier discusión acerca de la demanda de materia prima para toda clase de productos. La demanda de los productos forestales se deriva principalmente de la necesidad de combustible para calefacción y cocinar alimentos, papel para impresión y cartón, empaque y material de construcción. El consumo anual promedio mundial per cápita se ha calculado entre 0.6 y $0.7 \mathrm{~m}^{3}$ (FAO). En los Estados Unidos, el consumo per cápita es superior a los $2.3 \mathrm{~m}^{3}$ (USBC, 1990).

Según algunas fuentes (FAO), a nivel mundial, aproximadamente la mitad del consumo de madera se dedica a combustible. Esto coincide con la información presentada por la SEMARNAP para México, dentro del Programa Sectorial Forestal (1996). Según otros autores, en países en vías desarrollo "típicos", el consumo de madera para combustible llega al $70 \%$ del total (Salwasser et al., 1992). En cualquier caso, en esta clase de países, el consumo para combustible es alto, en proporción. En contraste, en Estados Unidos, que es el principal consumidor de madera en el mundo, el uso de madera como combustible es de $22 \%$, principalmente para usos industriales (Salwasser et al., 1992). El crecimiento de la población más que el incremento en consumo per cápita es la pricipal causa del aumento de la demanda mundial de productos forestales.

\section{IMPACTO DEL USO DE LOS RECURSOS FORESTALES EN EL AMBIENTE}

La preocupación contemporánea por el efecto invernadero, el calentamiento global, el debate sobre la sustentabilidad $y$ el impacto del hombre sobre la naturaleza han generado argumentos acerca de las bondades del uso de los recursos forestales en vez de materiales no renovables. Evaluar el impacto ambiental es un aspecto de suma importancia. Sin embargo, efectuar esta evaluación de una manera consistente y objetiva es una tarea difícil. En las últimas dos décadas, una forma de medición que ha venido cobrando auge es el proceso llamado "análisis de ciclo de vida" (LeVan, 1994). Este concepto no únicamente incluye el inventario de productos y materiales, como antes se 
consideraba en este tipo de análisis, sino también las fases de impacto ambiental y mejoramiento. Esta evaluación del impacto ambiental identifica y cuantifica la energía y materiales usados y los desperdicios arrojados al medio. Como todos los métodos, tiene sus aspectos benéficos y limitaciones, la principal de las cuáles es la dificultad de conseguir datos confiables y actualizados para hacer los análisis. La última etapa del ciclo de vida, el destino después de su uso inicial es difícil de estimar porque tiene lugar mucho después en el futuro y depende de la extensión de los programas de reciclaje en la comunidad.

En el Laboratorio de Productos Forestales de los Estados Unidos se está desarrollando un modelo analítico para evaluar el impacto del uso de los recursos sobre el medio ambiente de una manera integral, incluyendo consideraciones económicas, tecnológicas y ecológicas (LeVan, 1994). A este modelo lo denominan "Evaluación tecnológica ambiental". Modelos de este tipo parecen ser los más adecuados para hacer evaluaciones del impacto ambiental del uso de los recursos naturales, así como de sus beneficios económicos.

\section{VENTAJAS DEL APROVECHAMIENTO RACIONAL DE LOS RECURSOS FORESTALES}

Mi planteamiento, el cual coincide con el de los que propugnan un uso racional del bosque, es que el uso de la madera no es nocivo para el medio ambiente. En términos comparativos, la madera es uno de los materiales para construcción más benignos para el ambiente. Además, es virtualmente el único recurso natural renovable que es económicamente atractivo para usos estructurales y arquitectónicos, cuando menos en ambientes urbanos o rurales de los países desarrollados (Koch, 1992). Los materiales opcionales diferentes a los productos forestales para estos fines acero, aluminio y otros metales, concreto y plásticos- no son renovables (aunque algunos son reciclables).

Desde el punto de vista energético, el uso de la madera en la construcción es fundamental. Estudios en Dinamarca estiman que el sector de la construcción consume aproximadamente el $50 \%$ del total de la energía generada por el hombre. Los materiales sustitutos de la madera emplean considerablemente más energía por unidad de producción que la madera. Por ejemplo, en Estados Unidos, que es el país que más consume energía en el mundo, los productos a base de madera comprenden el $47 \%$ del total de las materias prima empleadas por la industria y consumen únicamente el $4 \%$ de la energía neta; en comparación, el acero aporta el $23 \%$ de la materia prima industrial y consume el $48 \%$ de la energía; para el cemento, las cifras correspondientes son de $20 \%$ (producción) y $8 \%$ (energía consumida) ("The truth...", 1987). $\mathrm{Si}$ las cifras de empleo de acero y cemento se incrementaran en sustitución de los recursos forestales, el aumento en el consumo de energía, con sus consecuencias ambientales y económicas negativas, sería sustancial. En el caso de México, los productos forestales no se emplean tan masivamente y en cambio, en el sector de la construcción, el acero y el cemento se usan preponderantemente. Si se revirtieran las proporciones de uso entre estos materiales, se lograrían ahorros energéticos significativos. En la Tabla 1 se muestran los datos de energía consumida y almacenada y emisión de bióxido de carbono durante la fabricación de una viga simplemente apoyada. 
Tabla 1. Energía consumida y almacenada y emisión de bióxido de carbono durante la fabricación de una viga simplemente apoyada (Natterer, s/f).

\begin{tabular}{||l|c|c|c|c||}
\hline MATERIALES & $\begin{array}{c}\text { ENERGIA } \\
\text { CONSUMIDA } \\
\left(\text { Julios } \times 10^{9}\right)\end{array}$ & $\begin{array}{c}\text { ENERGIA } \\
\text { ALMACENADA } \\
\left(\text { Julios } \times 10^{9}\right)\end{array}$ & $\begin{array}{c}\text { BIOXIDO DE } \\
\text { CARBONO } \\
\text { EMITIDO } \\
(\mathrm{kg})\end{array}$ & $\begin{array}{c}\text { BIOXIDO DE } \\
\text { CARBONO } \\
\text { ALMACENADO } \\
(\mathrm{kg})\end{array}$ \\
\hline Aluminio & 16 & 0 & 338 & 0 \\
\hline $\begin{array}{l}\text { Concreto } \\
\text { reforzado }\end{array}$ & 5 & 0 & 100 & 0 \\
\hline Acero & 3.8 & 0 & 76 & 0 \\
\hline $\begin{array}{l}\text { Madera } \\
\text { aserrada }\end{array}$ & 0.3 & 5 & 6.5 & 100 \\
\hline
\end{tabular}

Por otro lado, la madera como material de construcción tiene grandes ventajas. Como aislante, la madera también es más eficiente energéticamente que muchos sustitutos manufacturados, lo cual redunda en menor consumo energético durante la vida útil de una construcción (Salwasser et al., 1992). Los requerimientos energéticos para calentar o enfriar las viviendas dependen en gran medida de los materiales empleados para su construcción. Las estructuras de bastidores a base de madera son mucho mejor aislantes que las de bastidores de acero. Además, su eficiencia estructural en términos de la energía necesaria para producirla relativa al concreto, mampostería y acero es 15, 20 y 50 veces mayor, respectivamente, si se atienden únicamente aspectos de rigidez y, 25, 50 y 500 veces mejor si se considera la resistencia mecánica en compresión (Gordon, 1978).
Debido a todos estos factores, Koch (1992) estima que por cada mil millones de pies-tablón $(\mathrm{P}-\mathrm{T})^{2}$, (equivalentes a 2.36 millones de $\mathrm{m}^{3}$ ) de madera reemplazados por sustitutos manufacturados, el consumo anual de energía se incrementaría en aproximadamente 720 millones de galones (17.14 millones de barriles) de petróleo y las emisiones de bióxido de carbono se incrementarían en 7.5 millones de toneladas.

Adicionalmente, los bosques en crecimiento activo son almacenadores de carbono. Según Fosberg, citado por Salwasser et al. (1992), los bosques de Estados Unidos capturan el equivalente de aproximadamente el $9 \%$ del total de emisiones de bióxido de carbono. Por cada tonelada de madera que crece, un bosque joven produce 1.07 ton $y$ absorbe 1.47 ton de bióxido de carbono (The truth...", 1987). Ningún otro material provee este servicio ecológico que reduce el efecto de invernadero.

2 P-T. pie-tablón, la unidad de medida más usada en Norte-América, incluido México, equivale a un volumen de $30.5 \times 30.5 \times 2.54 \mathrm{~cm}$ 
Otro punto importante a considerar es el papel del bosque como el único elemento biosférico que regula la hidrología y la biogeoquímica terrestres. De ahí que los recursos suelo y agua también dependan de la cubierta forestal. En un país como México, con una gran extensión de suelo con vocación eminentemente forestal, esto es muy importante, sobre todo en vista de que gran parte del suelo forestal esta degradado.

El secreto para obtener todos estos beneficios esta en cultivar el bosque; utilizarlo para la producción de bienes de consumo, con un gasto energético reducido y hacerlo que crezca para que produzca mas oxígeno y capture mas bióxido de carbono al mismo tiempo. Por todos lados saldríamos ganando. Sin embargo, aunque la madera parece ser el material mas benigno para el medio ambiente, las preguntas que quedan por responder son, ¿qué tan bien manejado esta el inventario del bosque? y si, ¿están los recursos manejados de manera sustentable?

\section{CONCLUSIONES}

Parafraseando a Bowyer (1995), para resolver las necesidades de una población del doble de tamaño de la actual, la cual parece ser la máxima que la tierra puede sostener a largo plazo, sin afectar excesivamente el ambiente, se requerirá imaginación, un pensamiento realista y global, planeación cuidadosa e iniciativas audaces. Es obvio que no existen respuestas sencillas, si no que se deben atacar varios frentes de manera simultanea. Entre las principales iniciativas citadas por Bowyer (1991) y Koch (1992) se encuentran:
1) Se deben encontrar nuevas y mejores maneras de extraer, reducir y convertir las materias primas, incluyendo los recursos forestales, en artículos útiles, y rehusar los materiales y productos al máximo posible.

En el caso específicos de los recursos forestales, los esfuerzos de investigación deben dirigirse a incrementar el porcentaje del volumen cosechado de cada árbol convertido a bienes, estructurales y de otro tipo y, a prolongar la duración de la madera en servicio.

Una forma posible de aprovechar al máximo la madera y aumentar la eficiencia reduciendo el desperdicio, es a través de madera reconstituida la cual se elabora a base de fibras 0 astillas de la madera, de variadas formas y tamaños. De esta manera, se aprovecha mejor el recurso al emplear madera de menor calidad y tamaño, pues la de buena calidad y grandes dimensiones cada vez es mas escasa. En el país no se cuenta con mucha experiencia en el desarrollo de nuevos productos con material reciclado 0 con materias primas de baja calidad, excepto en el Departamento de Madera Celulosa y Papel de la universidad de Guadalajara, donde se han efectuado investigaciones en este tema (Fuentes, 1992; Pérez, 1994).

2) Deben buscarse mecanismos que faciliten la transparencia de tecnología de los países desarrollados a los menos desarrollados, para contribuir a un uso mas eficiente de los recursos a nivel mundial.

3) Las naciones con altos consumos per cápita, deben buscar maneras para reducir sus niveles de consumo. 
4) Deben mejorarse la eficiencia energética en la extracción, conversión a productos uso de las materias primas.

5) Deben establecerse mayor numero de plantaciones de árboles a nivel mundial donde se aplique manejo intensivo para producción de madera y de fibra de madera.

Bowyer (1995) concluye que lo que se requiere actualmente es mayor acción a gran escala mas que debate, lo cual me parece sensato, sin reconocer que no se puede dar por terminado el debate, ni ahora ni nunca. A este respecto, conviene anotar que en el Estado de Veracruz se han iniciado esfuerzos significativos para establecer plantaciones forestales con un horizonte a 39 años, dentro del marco del Plan Forestal Estatal 1995-2034. ("Plan..."). la importancia de las plantaciones tampoco escapa a las autoridades federales que la ubican como una de las primeras prioridades dentro de un Programa Sectorial Forestal ambicioso (SEMARNAP, 1996). En esta opinión coinciden otros especialistas (Gallegos y flores, citados por Jáuregui, 1994).

Otros investigadores (Evison y Horgan, 1992) destacan el echo de que el incremento al inventario mundial de madera comercial excede al nivel actual de consumo, lo cual en mi opinión, es motivo para conservar la calma y no alarmarse sobremanera acerca del futuro del bosque en el mundo. Esto, desafortunadamente no es uniforme en todo el planeta, pues mientras en muchos países, sobre todos los desarrollados, el bosque crece, en otros, menos afortunados, el recurso forestal disminuye, aquí si, de manera alarmante. Lamentablemente, este también es el caso de nuestro país (Jáuregui, 1994). Con todo, creo que en México todavía existe margen de maniobra, pero no por mucho tiempo; por eso la adopción de programas de conservación y aprovechamiento de los recursos forestales, que sean realistas y eficaces y sobre todo, pensados para el largo plazo, es vital.

No únicamente los seres humanos nos veremos afectados por el incremento en las emisiones de bióxido de carbono en la atmósfera; la flora y la fauna también resentirán las consecuencias. Deben intensificarse los esfuerzos para informar a la sociedad a cerca de los beneficios de los bosques manejados intensamente en capturar el bióxido de carbono, además de ilustrarlos de la conveniencia de reducir la extracción y consumo de combustibles fósiles y materiales no renovables. Nuestro país no puede darse el lujo de desperdiciar un recurso tan valioso y tan necesario para su bienestar. En esta labor, todos tenemos la palabra. Hagámosla oír.

\section{REFERENCIAS}

Bowyer, J. 1991. Global climate change, material needs and environmental quality. 1991 Conference on forests and global change, Arlington, Va.

Bowyer, J. 1995. Wood and other raw materials for the $21^{\text {st }}$ Century. Forest Products Journal 45(2):17-24.

Cleveland, C. J., R. Constanza, C.A.S. Hall y R. Kaufmann. 1984. Energy and the U.S. economy: A biophysical perspective. Science (225):890-897, 31 agosto de 1984.

Evison, D. y Horgan, G. 1992. Competition for New Zealand Forestry Products. Reprint 2471, Forest Research Institute, Rotorua, Nueva Zelanda 
FAO. 1960-1989. FAO Yearbook of Forest products, varias ediciones. FAO Wood and Wood Products 1961-1989 to 2010.

Fuentes T., F.J., Silva G., J.A., Rodríguez A., R. y Bravo G., L.R. 1992. Tableros aglomerados de materias primas no convencionales. Ciencia y Desarrollo 17(102):106-126.

Gordon, J. E. 1978. Structures and why things don't fall down. Plenum Press. New York.

Jáuregui, F. 1994. La industria maderera rezagada tecnológicamente. Revista Vinculación Universidad - Sociedad, Universidad de Guadalajara, p:5-11, julio de 1994.

Koch, P. 1992. Wood versus nonwood materials in U.S. residential construction: some energy-related global implications. Forest products journal 42(5):31-42.

LeVan, S. L. 1994. Life cycle analysis. Annual Proceedings of the Canadian Wood Preservation Association, Toronto, Canadá.

Natterer, J. S/F. Energy criteria for timber structures. Datos sin publicar.
Plan Sectorial Forestal del Estado de Veracruz (1996-2034). Gobierno del Estado de Veracruz y Secretaría de Medio Ambiente, Recursos Naturales y Pesca.

Pérez V., I. 1994. Desechos para construir casas y muebles. Revista Vinculación Universidad - Sociedad, Universidad de Guadalajara, p:12-16, julio de 1994.

Salwasser, H., MacCleery, D. W. y Snellgrove, T. 1992. A perspective on people, wood, wildlife, and environment in U.S. forest stewardship. USDA Forest Service. Presentado en la Comisión Forestal de América del Norte, en Cancún, México.

SEMARNAP, 1996. Programa Sectorial Forestal 1995-2000. Subsecretaría de Recursos Naturales. México, D.F. 10 de Enero de 1996.

The truth about American Forests (Evergreen), 1987.

USBC, 1990. U.S. Bureau of Census Statistical Abstract of the United States: $1990.110^{\text {th }}$ ed. Washington, D.C. Tablas 1176 y 1177. 